Classification

Physics Abstracts

61.30

\title{
Molecular arrangement of mesogenic disc-like compounds : the hexa-n-alkoxy and hexa-n-alkanoyloxy triphenylenes
}

\author{
M. Cotrait, P. Marsau, M. Pesquer ( $\left(^{\dagger}\right)$ and V. Volpilhac \\ Laboratoire de Cristallographie et de Physique Cristalline (*), Université de Bordeaux I, \\ 351, Cours de la Libération, 33405 Talence Cedex, France \\ (†) Laboratoire de Chimie Physique (**), Université de Bordeaux I, 351, Cours de la Libération, \\ 33405 Talence Cedex, France
}

(Reçu le 16 juillet 1981, accepté le 29 septembre 1981)

\begin{abstract}
Résumé. - L'étude théorique de séries homologues d'hexaesters et d'hexaéthers du triphénylène, molécules discoïdes mésomorphogènes a été effectuée par des méthodes semi-empiriques. Les conformations préférentielles d'une molécule isolée, d'une paire de molécules et d'un ensemble de molécules ont été établies.

$\mathrm{Au}$-delà du terme butylique, les hexaesters forment des colonnes irrégulières, résultant de l'empilement de paires, avec des distances $d$ et $d^{\prime}$ de 3,75 et 4,45 $\AA$ entre plateaux aromatiques parallèles successifs. Les hexaéthers, par contre, forment des colonnes régulières $(d \simeq 3,28 \AA)$ de type hélicoïdal de pas égal à $14 \AA$.
\end{abstract}

Les résultats sont en bon accord avec ceux déduits de la diffraction des rayons $\mathrm{X}$ par la mésophase.

\begin{abstract}
A theoretical study of homologous series of triphenylene hexaesters and hexaethers, disc-like mesogenic molecules, has been undertaken with semi-empirical methods. Preferential conformations of an isolated molecule, a pair of molecules and a set of molecules have been established.

For butyl and further homologues, hexaesters give irregular columns, resulting in piled pairs, with distances $d$ and $d^{\prime}$ equal to 3.75 and $4.45 \AA$ between neighbouring aromatic cores. On the other hand hexaethers give regular columns $(d \simeq 3.28 \AA)$ of helicoidal type with a pitch equal to $14 \AA$.

These results are in agreement with data from X-ray diffraction on the mesophase.
\end{abstract}

Introduction. - It is a well-known fact that disc-like molecules are able to give a thermotropic mesomorphic phase called discotic mesophase, which looks very different from nematic or smectic phases [1-5] and for which classifications have been proposed $[6,7]$.

Among the first molecules which have been found to show a discotic mesophase are the hexasubstituted triphenylene derivatives $[2,8,9]$, whose general formula is :

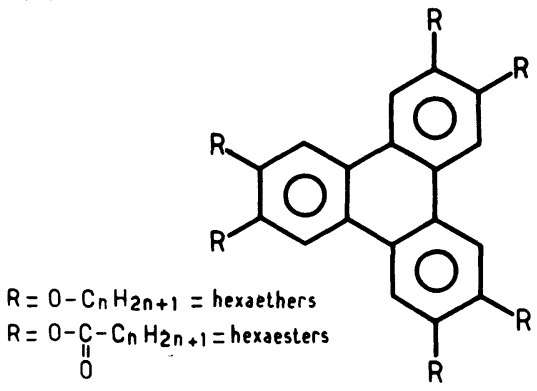

(*) LA 144 C.N.R.S.

(**) ERA 312.
Three homologous series have been synthetized up to now with :

$\mathrm{R}=\mathrm{O}-\mathrm{C}_{n} \mathrm{H}_{2 n+1}$ : hexa-n-alkoxy triphenylene (hexaethers),

$\mathrm{R}=\mathrm{O}-\mathrm{CO}-\mathrm{C}_{n} \mathrm{H}_{2 n+1}$ : hexa-n-alkanoyloxy triphenylene (hexaesters),

$\mathrm{R}=\mathrm{O}-(\mathrm{CO})-\mathrm{C}_{6} \mathrm{H}_{4}-\mathrm{C}_{n} \mathrm{H}_{2 n+1}$ : hexa-n-alkoyl (or alkoxy) benzoates of triphenylene (hexabenzoates) for different lengths of the aliphatic chains. The hexaethers, hexaesters and hexabenzoates show a crystal-to-mesomorphic transition respectively for $n \geqslant 4, n \geqslant 6$ and $n \geqslant 5$ [10]. Thermodynamic properties (temperature and heat of transition) and optical textures have been extensively studied $[8,11]$. In a recent paper we presented the crystalline arrangement for three triphenylene hexaesters with propyl, butyl and pentyl chains. Because of a very high thermal scattering and molecular disorder, only the crystal structure of the propyl derivative could be solved [12]. The resolution was unfortunately rather low $(R=0.37)$ and the positions of alkyl chains could not be assigned with precision. This is the 
reason why we entered upon a study of this compound by semi-empirical calculations [13], which established the preferential conformations for the isolated molecule and the association of molecules in stable pairs.

Encouraged by this result we decided to go further to undertake a more general study :

a) on homogeneous series of triphenylene hexaesters and hexaethers with $n$ running from 2 to 7 for the former series and from 3 to 8 for the second one;

b) with an increased number of molecules, in order to simulate the molecular arrangement in the mesophase.

1. Methodology. - Bond lengths and angles used in this paper result from mean values observed in crystal structures of molecules having common chemical groups with the present ones [14].

The calculation of conformational energy has been performed by using semi-empirical methods described by Scheraga [15].

For each conformation the total energy $E_{\mathrm{T}}$ is calculated as :

$$
E_{\mathrm{T}}=E_{\mathrm{VW}}+E_{\mathrm{el}}+E_{\mathrm{rot}}
$$

where $E_{\mathrm{vW}}, E_{\mathrm{el}}$ and $E_{\mathrm{rot}}$ are respectively the Van der Waals, electrostatic, and torsional contributions.

The Van der Waals term has been calculated using the well-known Lennard-Jones function :

$$
E_{\mathrm{VW}}\left({ }^{1}\right)=\sum_{i j}\left[-\frac{A_{i j}}{\left(r_{i j}\right)^{6}}+\frac{B_{i j}}{\left(r_{i j}\right)^{12}}\right]
$$

where $r_{i j}$ is the distance between $i$ and $j$ atoms, $A_{i j}$ and $B_{i j}$ are coefficients which depend on the nature and the hybridation states of $i$ and $j$ atoms.

The values used here are those of Scheraga [16] rather than Hopfinger's [17]. As a matter of fact Scheraga's coefficients have been tested from a large number of molecules including aromatic polycyclic rings.

The electrostatic contribution is given by the relationship :

$$
E_{\mathrm{el}}\left({ }^{1}\right)=\sum_{i j} 332 \frac{q_{i} q_{j}}{r_{i j}},
$$

where $q_{i}$ and $q_{j}$ are the partial charges centred on non-bonded $i$ and $j$ atoms calculated by the CNDO/2 method, with the formalism and parameters of Pople and Beveridge [18] : 332 is a conversion factor to get $E_{\mathrm{el}}$ in $\mathrm{kcal}_{\text {.mole }}{ }^{-1}$ : the dielectric constant has been taken equal to 2 .

The rotational energy is calculated through the following formula :

$$
E_{\mathrm{rot}}\left({ }^{1}\right)\left(\theta_{i}\right)=\frac{1}{2} E_{\max }\left(\theta_{i}\right)\left(1 \pm \cos n \theta_{i}\right)
$$

(') All energy terms are expressed in kcal.mole ${ }^{-1}$. where $E_{\max }\left(\theta_{i}\right)$ is the barrier height and $n$ the periodicity of the function. $E_{\max }\left(\theta_{i}\right)$ values used here come from experimental values for similar chemical groups.

For the rotation around the $\phi-\mathrm{O}$ bond in both series $E_{\max }\left(\theta_{i}\right)$ is close to $0.5 \mathrm{kcal} . \mathrm{mole}^{-1}$ [19]; a similar value has been adopted for the rotation around the $\mathrm{C}(\mathrm{O})-\mathrm{CH}_{2}$ in hexaesters [20]. These rotations barriers are rather low. On the other hand the rotations around $\mathrm{O}-\mathrm{CO}$ in hexaesters, $\mathrm{O}-\mathrm{CH}_{2}$ in hexaethers and $\mathrm{CH}_{2}-\mathrm{CH}_{2}$ in both series are much higher : $8.75,3.7$ and $2.7 \mathrm{kcal}^{\text {mole }}{ }^{-1}$ respectively, and are not allowed to vary [20-22].

2. Conformations of isolated molecules. - Because hexaether and hexaester molecules have a threefold symmetry and the chains branched on different benzene rings are far away, the conformational study may be restricted to one third of the molecules. So there are two independent chains called I and II, whose movements can be described by four torsional angles for hexaesters : $\theta_{1}, \theta_{2}, \theta_{1}^{\prime}$ and $\theta_{2}^{\prime}$ and two torsional angles for hexaethers : $\theta_{1}$ and $\theta_{1}^{\prime}$ (see Figs. $1 a$ and $1 b$ ). They are defined as follows : $\theta_{1}\left(\theta_{1}^{\prime}\right)$ correspond to the $\left[\mathrm{C}_{\mathrm{IV}}-\mathrm{C}_{\mathrm{I}}-\mathrm{O}_{1}-\mathrm{C}_{0}\right]$ and $\left[\mathrm{C}_{\mathrm{III}}-\mathrm{C}_{\mathrm{II}}-\mathrm{O}_{1}^{\prime}-\mathrm{C}_{0}\right]$ dihedral angles for both series; $\theta_{2}\left(\theta_{2}^{\prime}\right)$ correspond to
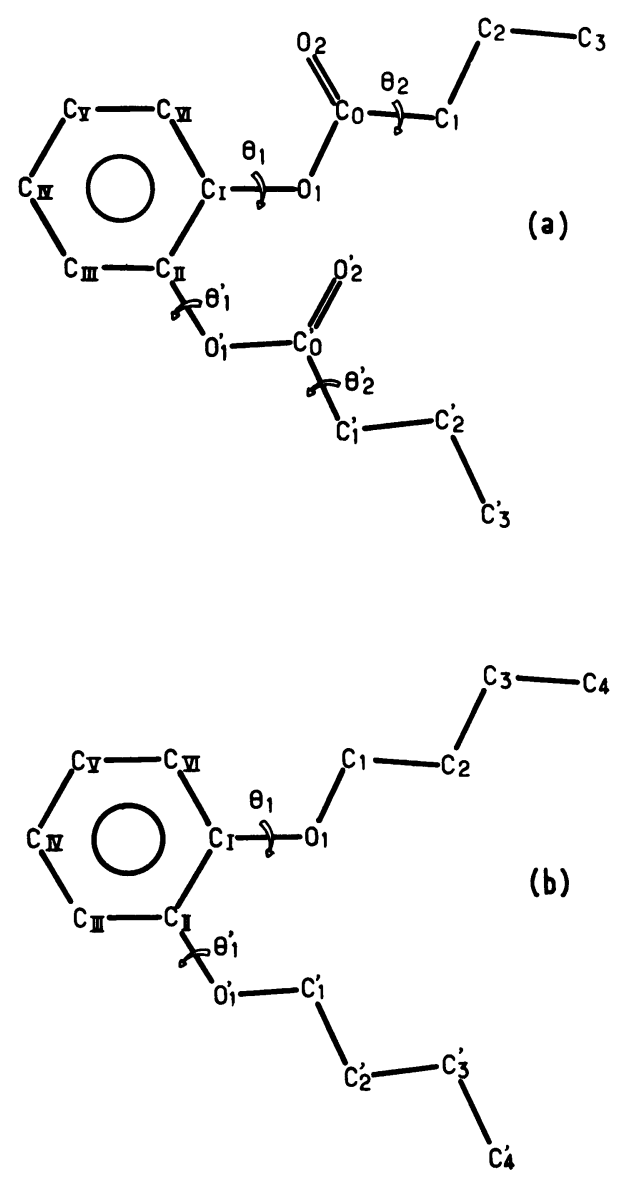

Fig. 1. - Schematic representation of one third of hexapropylester $(a)$ and hexabutylether (b) molecules, with torsional angles. Hydrogen atoms have been omitted. 
the $\left[\mathrm{O}_{1}-\mathrm{C}_{0}-\mathrm{C}_{1}-\mathrm{C}_{2}\right]$ and $\left[\mathrm{O}_{1}^{\prime}-\mathrm{C}_{0}^{\prime}-\mathrm{C}_{1}^{\prime}-\mathrm{C}_{2}^{\prime}\right]$ dihedral angles for hexaesters only; a positive value is relative to a clockwise rotation around a given bond. The total energy $E_{\mathrm{T}}$ is minimized in the following way :

$\theta_{1}$ is varied while the other angles are fixed; the energy $E_{\mathrm{T}}$ varies with $\theta_{1}$ ' and reaches a minimum for some $\theta$ value, let us say $\theta_{1 \min } ; \theta_{1}$ is then fixed to its minimum value and $\theta_{2}$ varied, while $\theta_{1}^{\prime}$ and $\theta_{2}^{\prime}$ are invariant. This procedure is successively used for all torsional angles. Angular steps are taken equal to $1^{\circ}$.

As far as hexaesters are concerned the most stable conformations have been obtained for $\theta_{1}=\theta_{1}^{\prime} \simeq-55^{\circ}$; $\theta_{2}=\theta_{2}^{\prime} \simeq-75^{\circ}$ for all lengths $(n=2$ to 7$)$ of the alkyl chains $\left({ }^{2}\right)$. These values are relative to a confi-
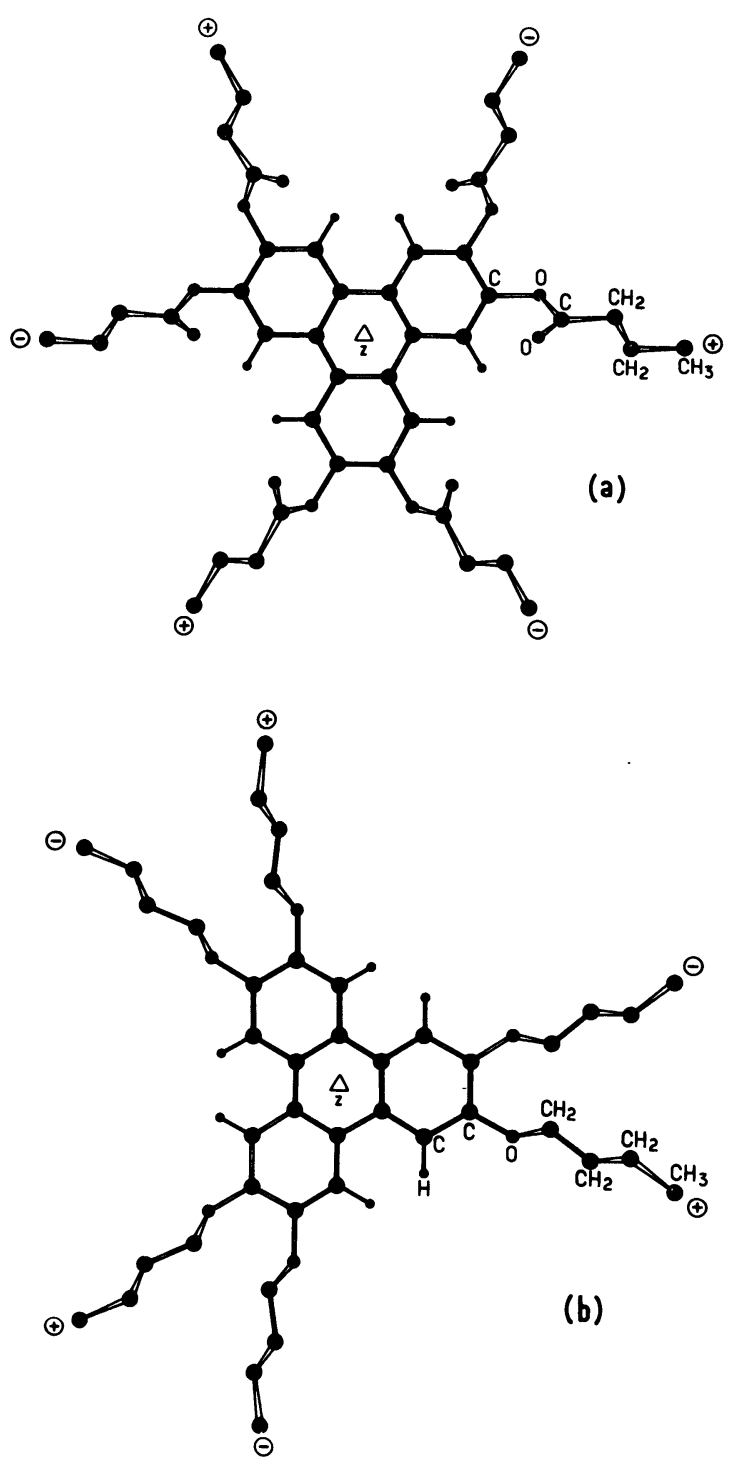

Fig. 2. - The most stable conformation of hexapropylester $(a)$ and hexabutylether $(b)$ molecules. The $(+)$ and $(-)$ signs are relative to chains directed upwards and downwards respectively.

$\left({ }^{2}\right)$ Let us recall that for the triphenylene propyl ester the values are $\theta_{1}=\theta_{1}^{\prime}=-56^{\circ}, \theta_{2}=\theta_{2}^{\prime}=-77^{\circ}$ [13]. guration where the chains I and II are respectively situated above and under the triphenylene ring (configuration (i)), the ester $\mathrm{O}-\mathrm{CO}-\mathrm{C}_{1}$ group making an angle close to $-60^{\circ}$ with the triphenylene core.

In the case of hexaethers the most stable conformations correspond to $\theta_{1}=\theta_{1}^{\prime} \simeq-122^{\circ}$ for all lengths $(n=3$ to 8$)$ of the alkyl chains. These values are relative to the configuration (i), the mean plane of the alkyloxy chain making an angle close to $+60^{\circ}$ with the aromatic ring.

The most stable conformations are shown in figure $2 a$ and figure $2 b$ respectively for hexaesters and hexaethers.

3. Conformation of dimers. - The conformational study of dimers has been performed in the following way :

- molecules are supposed to be in their most stable conformation;

- they have the same threefold axis;

- they are allowed to move along it and to rotate around it.

These restrictions are justified from experimental results : X-ray diffraction studies both on crystal and on the mesophases show that triphenylene cores are parallel and inclined to give columns. Consequently the energy is a function of the distance $d$ between the aromatic rings and the rotational angle $\alpha$ around their common axis $\mathrm{Oz}\left({ }^{3}\right)\left(\alpha=0^{\circ}\right.$ when rings are superposed along $\mathrm{Oz}$ ).

Translational and angular steps are taken equal to $0.01 \AA$ and $1^{\circ}$ respectively.

3.1 HeXAESTERS. - The most stable conformation is obtained for :

$d=3.63 \AA$ and $\alpha=-30^{\circ}$ (or $90^{\circ}$ ) for propyl and butyl chains $\left(\mathrm{C}_{3}, \mathrm{C}_{4}\right.$ derivatives).

$d=3.74 \AA$ and $\alpha=-20^{\circ}$ (or $100^{\circ}$ ) for longer chains $\left(\mathrm{C}_{5}, \mathrm{C}_{6}\right.$ and $\mathrm{C}_{7}$ derivatives).

This evolution when going from $\mathrm{C}_{4}$ to $\mathrm{C}_{5}$ is due to steric hindrance between hydrogen atoms of the aliphatic chains when their length increases.

The conformation of the propyl $\left(\mathrm{C}_{3}\right)$ derivative dimer is shown in figure $3 a$. A similar configuration is observed for longer chains, for instance for the $\mathrm{C}_{5}$ derivative. The relative disposition of the two molecules in a pair is very special. The carbonyl oxygen of one molecule is facing two contiguous hydrogens of the other molecule with rather short $\mathrm{H}$...O distances of : $2.12 \AA$. This configuration stabilizes a great deal the formation of the dimer. The energy rises sharply when $\alpha$ moves away from the minimum value, as shown in figure $4 a$; for $\Delta E=0.6 \mathrm{kcal}$. mole ${ }^{-1}(\mathrm{RT})$ : $\delta \alpha \simeq 2^{\circ}$ and $\delta d=0.3 \AA$. The dimer can be considered as rigid except for the alkyl chains.

$\left({ }^{3}\right)$ A positive value is relative to a clockwise rotation around the $\mathrm{Oz}$ axis. 


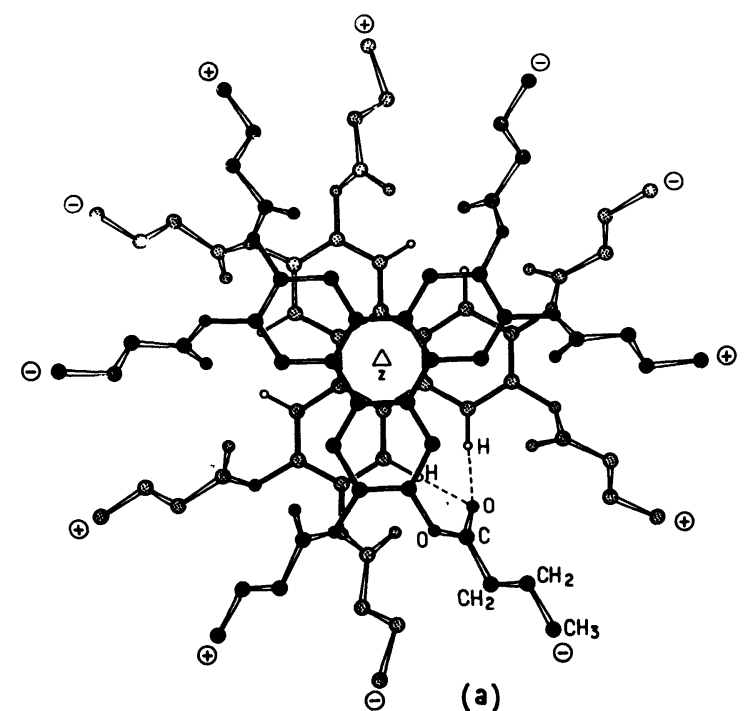

(a)

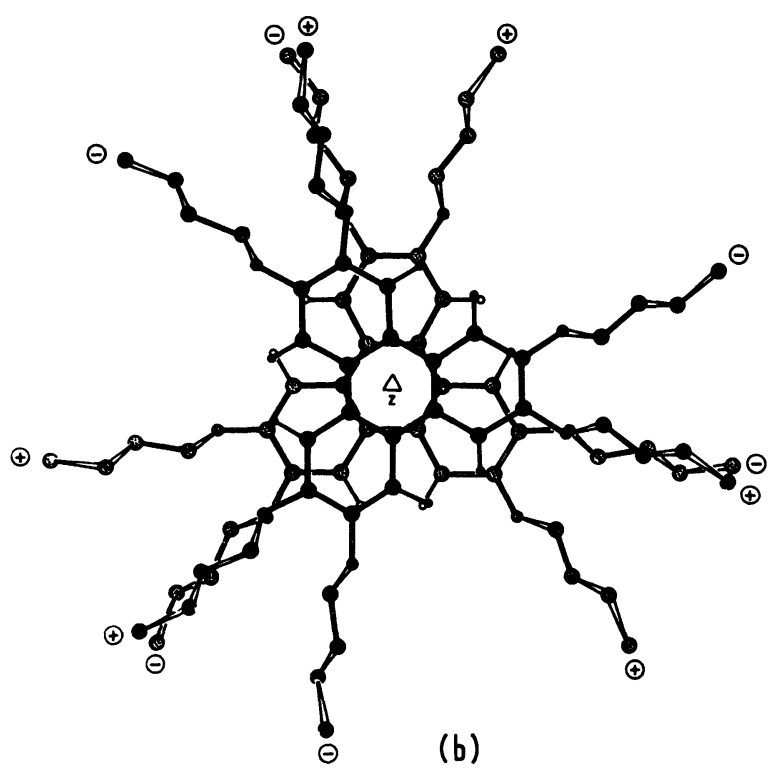

Fig. 3. - The most stable arrangement of hexapropylester (a) and hexabutylether (b) molecules into dimers.

3.2 HeXAETHERs. - The most stable conformation is obtained for : $d \simeq 3.27$ and $\alpha=-27^{\circ}$ for all derivatives from $\mathrm{C}_{3}$ to $\mathrm{C}_{8}$. The $d$ distance is somewhat smaller than for hexaesters. This can of course be attributed to the lower steric volume of the ether group. The conformation of the dimer in the case of the butyl ether is shown in figure $3 b$. The energy varies more slowly with $\alpha$ than in the hexaesters for

$$
\Delta E=0.6 \mathrm{kcal} . \mathrm{mole}^{-1}: \delta \alpha \simeq 5^{\circ}, \quad \delta d \simeq 0.2 \AA .
$$

4. Molecular packing of several molecules. - The conformational study of a set of molecules has been performed in the same way as for dimers :

- Dimers are supposed to be in their most stable conformation, as seen before.
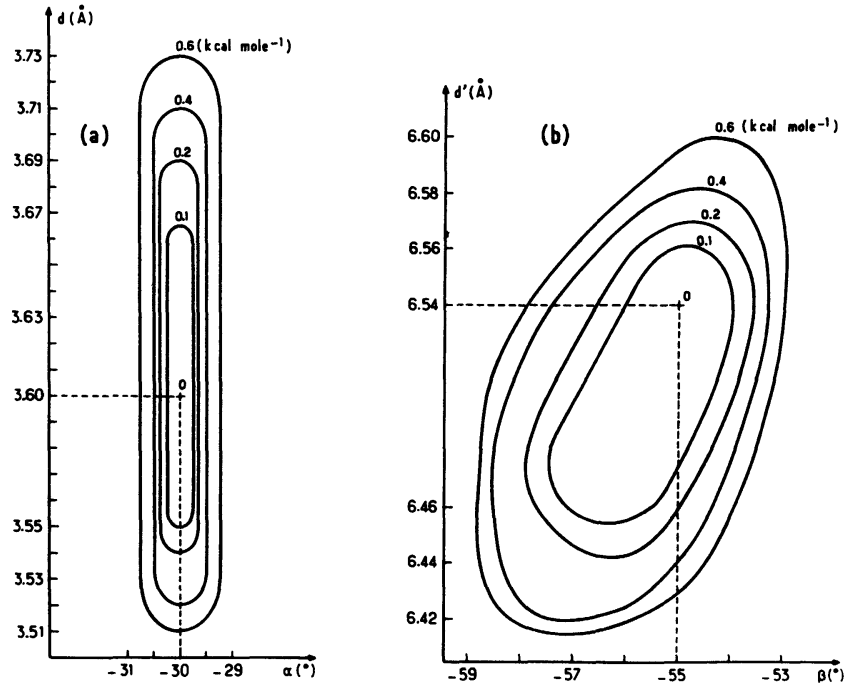

Fig. 4. - Energy levels (kcal.mole $\left.{ }^{-1}\right):(a)$ versus $d$ and $\alpha$ for the hexapropylester molecule and (b) versus $d^{\prime}$ and $\beta$ for the hexabutylether-dimer.

- They have the same threefold axis $\mathrm{Oz}$.

- They are allowed to move along it and to rotate around it.

These restrictions are justified for the same reasons as for the dimer. The energy is then a function of the distance $d^{\prime}$ between the centre of each pair and the rotational angle $\beta$ around their common axis $\left(\beta=0^{\circ}\right.$ when triphenylene rings 1 and 3 are superposed in projection along $\mathrm{Oz}$ ).

4.1 HeXaesters. - The most stable conformation of a tetramer is obtained for :

$-d^{\prime}=7.24 \AA$ and $\beta=-60^{\circ}$ (or $60^{\circ}$ ) for $\mathrm{C}_{3}$ and $\mathrm{C}_{4}$ derivatives. As $d^{\prime} \simeq 2 d$ and $\beta \simeq 2 \alpha$, the arrangement in a column is a regular one of helical type.

$-d^{\prime}=8.24 \AA$ and $\beta=4^{\circ}$ for $C_{5}$ to $C_{7}$ derivatives.

The distance $\left(d^{\prime}-d\right)$ and the angle $(\beta-\alpha)$ between adjacent molecules of the two pairs are then equal to $4.5 \AA$ and $24^{\circ}$ respectively.

The molecular arrangement in a column is irregular with a mean spacing around $4.12 \AA$ (Fig. 5).

This result is in agreement with the X-ray diffraction experiment on the $\mathrm{C}_{7}$ and $\mathrm{C}_{11}$ mesophases : irregular columns with disordered chains with an average intermolecular distance of $4 \AA$ along the axis [23].

4.2 HeXAethers. - The most stable conformation of the tetramer is obtained for : $d^{\prime} \simeq 6.54 \AA$ and $\beta \simeq-55^{\circ}$ (for all derivatives $\left(C_{3}\right.$ to $\left.C_{8}\right)$ ) $\left({ }^{4}\right)$. As $d^{\prime}=2 d$ and $\beta=2 \alpha$, the arrangement in a column is a regular one with helical type (see Fig. 5). Taking

$\left({ }^{4}\right)$ For $\Delta E=0.6$ kcal.mole ${ }^{-1}$ (RT) : $\delta \beta \simeq 6^{\circ}$ and $\delta d^{\prime} \simeq 0.2 \AA$ as shown in figure $4 b$. 


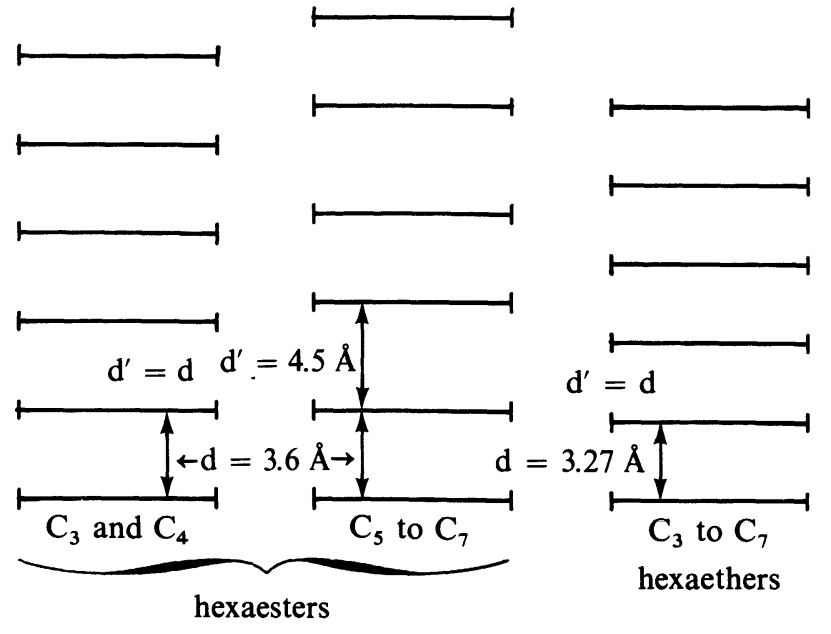

Fig. 5. - Schematic representation of molecular stacking for both hexaesters and hexaethers.

into account the threefold symmetry, the helix pitch corresponding to an $\alpha$ rotation of $120^{\circ}$ is close to $14 \AA$.

This is in a rather good agreement with X-ray diffraction on the $\mathrm{C}_{5}, \mathrm{C}_{7}$ and $\mathrm{C}_{8}$ mesophases: regular columns with regular spacing equal to $3.6 \AA$ and helicoidal arrangement which is close to $13 \AA$ [24] (Fig. 5).

The small differences in observed and calculated values can be accounted for by the fact that chains have been considered as rigid.

For both hexaesters and hexaethers the small discrepancies between observed and calculated values can be accounted for by the following approximation : the chains have been considered as rigid, in their most stable conformation for the isolated molecules, when approaching molecules and this is not true. A more sophisticated approach taking into account conformational freedom of alkyl chains would have been time consuming and very expensive.

5. Influence of neighbouring columns. - Neighbouring columns are in contact through the end of their aliphatic side-chains; interactions between them should be weak because they are due to Van der Waals forces. Moreover, X-ray diffraction pattern on the mesophases shows that there are no correlations between these columns [23, 24].

6. Conclusion. - The molecular arrangement of some disc-like compounds (triphenylene hexaesters and hexaethers), obtained from semi-empirical calculations is in relatively good agreement with experimental results despite various approximations made in the theoretical approach.

This is rather interesting because :

1) The structure of the mesophase can be explained in terms of intermolecular interactions. In the present case the reason why hexaesters and hexaethers give different arrangements can be correlated to their molecular structure.

2) The evolution, if any, of the mesophase structure with the lengths of alkyl chains can be interpreted at the atomic level (steric hindrance).

\section{References}

[1] Chandrasekhar, S., Sadashiva, B. K., Sureh, K. A., Pramana 9 (1977) 471.

[2] Billard, J., Dubois, C., Nguyen, H. T., ZanN, A., Nouv. J. Chim. 2 (1978) 535.

[3] Queguinier, A., Zann, A., Dubois, J. C., Billard, J., Proc. Int. Liquid Cryst. Conf., Bangalore (1979) (Meyden and Son, London).

[4] Destrade, C., Malthete, J., Nguyen, H. T., GaspaRoux, H., Phys. Lett. 78A (1980) 82.

[5] Nguyen, H. T., Destrade, C., Gasparoux, H., Phys. Lett. 72A (1979) 251.

[6] Destrade, C., Bernaud, M. C., Gasparoux, H., Levelut, A. M., Nguyen, H. T., Proc. Int. Liq. Cryst. Conf., Bangalore (1979) (Meyden and Son, London).

[7] Billard, J., Proceedings of the Garmisch-Partenkirchen Liq. Cryst. Conf. (1980) 383.

[8] Destrade, C., Mondon, M. C., Malthete, J., J. Physique Colloq. 40 (1979) C3-17.

[9] Nguyen, H. T., Destrade, C., Gasparoux, H., Phys. Lett. 72A (1979) 251.

[10] Mondon-Bernaud, C., Thesis $n^{0} 1580$ (1980), Bordeaux and references therein.

[11] Destrade, C., Mondon-Bernaud, M. C., Nguyen, H. T., Mol. Cryst. Liq. Cryst. 49 (1979) 169.

[12] Cotrait, M., Marsau, P., Destrade, C., MalTHETE, J., J. Physique Lett. 40 (1979) L-519.
[13] Pesquer, M., Cotrait, M., Marsau, P., Volpilhac, V., J. Physique 41 (1980) 1039.

[14] KennaRD, O., Watson, D. G., Molecular structure and Dimensions, Utrecht-Oostweek, 1972.

[15] Scheraga, H. A., Adv. Phys. Org. Chem. 6 (1968) 103.

[16] Momany, F. A., Carruthers, M. L., MacGuire, R. F., Scheraga, H. A., J. Phys. Chem. 78, 6 (1974) 1595.

[17] Hopfinger, A. J., Conformational properties of macromolecular (New York, Acad. Press) 1973, p. 47.

[18] Pople, J. A., Beveridge, D. L., Approximative Molecular Orbital Theory (Mac Graw Hill, New York) 1970.

[19] Perez, S., Saint-Pierre, J., Marchessault, R. H., Can. J. Chem. (1978) 2866.

[20] Yan, J. F., Van Der Kool, G., Scheraga, H. A., J. Chem. Phys. 49 (1968) 2713.

[21] Perricaudet, M., Pullman, A., Int. J. Pept. Protein Res. 5 (1973) 99.

[22] HerschBACH, D. R., Bibliography for hindered internal rotation and Microwave Spectroscopy, Lawrence Radiation Lab., Univ. of California, 1962.

[23] Levelut, A. M., Proc. Intern. Conf. on Liquid Crystals, Bangalore edited by S. Chandrasekhar (Heyden and Son, London) 1980, p. 21.

[24] Levelut, A. M., J. Physique Lett. 40 (1979) L-81. 